\title{
Fourier-like Thermal Relaxation of Nanoscale Explosive Hot Spots
}

\author{
Matthew P. Kroonblawd, ${ }^{*} \dagger$ Brenden W. Hamilton ${ }^{\ddagger}{ }^{\ddagger}$ and Alejandro Strachan ${ }^{\ddagger}$ \\ $\dagger$ †hysical and Life Sciences Directorate, Lawrence Livermore National Laboratory, \\ Livermore, CA 94550, United States \\ $\ddagger$ School of Materials Engineering and Birck Nanotechnology Center, Purdue University, \\ West Lafayette, Indiana 47907, United States \\ E-mail: kroonblawd1@llnl.gov
}




\begin{abstract}
Hot spots are local regions of high temperature that are widely considered to govern explosive initiation. Hot spot dynamics rests on a delicate balance between heat generation due to chemical reactions and heat loss through thermal conduction, making accurate determinations of the conductivity under extreme conditions a key component of predictive explosive models. We develop here an approach to directly determine the thermal transport properties of explosive hot spots with realistic initial structures through a combination of molecular dynamics (MD) and diffusive heat equation (HEq) modeling. Effective thermal conductivity values are determined by fitting HEq models to MD predictions of long timescale hot spot relaxation. The approach is applied to model hot spots in the molecular crystalline explosive TATB for a range of shock strengths and two limiting cases for impact orientation. Isotropic and anisotropic HEq models yield similar results, despite TATB exhibiting some of the largest and most anisotropic thermal conductivity values for an explosive near normal conditions. The conductivity is found to be a strong function of density, which parametrically captures dependence on temperature, pressure, and material state. The associated root-mean square errors of the fitted HEq models are approximately $5 \%$ of MD predicted final equilibrium temperatures. Conductivity values determined here for TATB hot spots are considerably larger than those used in a prior hot spot criticality study, which may significantly impact predictions for critical hot spot sizes. The approach provides a convenient foundation for determining the effective thermal conductivity for hot spot problems in other explosives and directly yields information on reasonable approximations that might be taken in higher-level models for those materials.
\end{abstract}

\title{
1 Introduction
}

Thermal transport is widely recognized as one of the dominant physics terms influencing the shock initiation and detonation properties of energetic materials. ${ }^{1}$ Explosive initiation is considered to be controlled through hot spots, ${ }^{2-4}$ which are regions of high temperature, 
pressure, and strain that form from the shock-induced collapse of microstructural pores. A critical hot spot is one in which heat generation due to exothermic chemistry outweighs heat loss to the surroundings from conductive dissipation and initial endothermic chemical decomposition processes. ${ }^{5}$ Critical hot spots form steady deflagration (burn) waves ${ }^{6}$ that may interact ${ }^{7}$ and ultimately coalesce in a detonation front if they occur in sufficient density. This makes thermal conductivity a key parameter in coarse-grained models of explosives. ${ }^{8}$ Understanding the thermal transport properties of explosive hot spots is thus a necessary aspect for developing predictive initiation and detonation models given the significant bearing they have on the ensuing sequence of events.

Considerable effort has been spent to determine the anisotropic thermal conductivity of single crystals of a range of explosive materials, including hexahydro-1,3,5-trinitro-s-triazine (RDX), ${ }^{9-12}$ octahydro-1,3,5,7-tetranitro-1,3,5,7-tetrazocine (HMX), ${ }^{13,14}$ and 1,3,5-triamino2,4,6-trinitrobenzene (TATB). ${ }^{15-19}$ Most all of the experimental determinations of thermal conductivity for these materials were performed on polycrystalline pressed powder samples (see discussions in Refs. 12, 14, and 15). The focus on limiting cases ranging from (near) perfect single crystals to powders that are rife with defects neglects the fundamental role that material structure plays in determining the thermal conductivity.

It is well known that the thermal conductivity is reduced by a range of factors that limit the mean free path length of phonons, the primary energy carrier in these electrically insulating materials. ${ }^{20,21}$ These limiting factors include scattering at crystal defects such as vacancies and grain boundaries, phase boundaries, and the size scale for regions with large temperature gradients (e.g., hot spot size) to name a few cases that are relevant to explosive hot spots. Phonon transport can exhibit a range of diffusive and ballistic-like characteristics over a range of length scales as the phonon spectrum exhibits a range of mean free path lengths. ${ }^{22,23}$ Phonon motion is ballistic (i.e., unscattered) over length scales that are smaller than the mean free path and thus do not contribute to the apparent thermal conductivity (i.e., when Knudsen number »1). Thermal conductivity is an inverse measure of resistance 


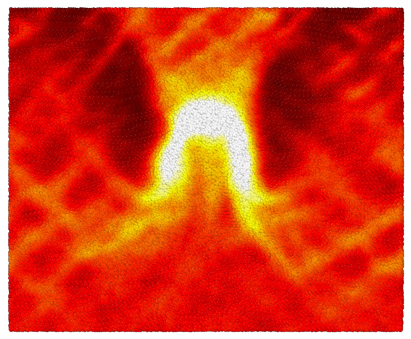

Temperature (K)

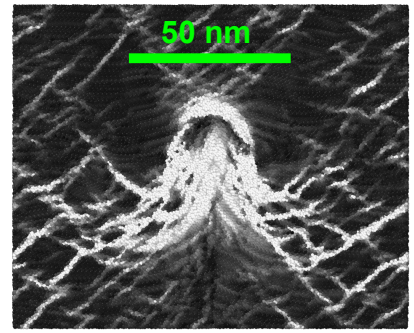

Shear Strain

300

$0 \longdiv { 1 + }$

Figure 1: Example temperature and shear strain fields of a TATB hot spot formed from shock-induced pore collapse. The strain field was computed based on molecular centers of mass with respect to the pre-collapse configuration using OVITO. ${ }^{25}$ The shear strain is unitless and should be interpreted qualitatively as the precise numerical value depends on parameters in the analysis. ${ }^{26}$ This hot spot was generated for a shock along $\mathbf{N}_{(001)}$ with a $2.0 \mathrm{~km} \cdot \mathrm{s}^{-1}$ impact speed and is discussed in detail below.

to energy flow that only accounts for those modes that exhibit diffusive motion arising from many resistive scattering events. Simple theoretical treatments such as Matheissien's rule assume that phonon scattering mechanisms contribute independently, leading to a relation of the effective mean free path (and thus the effective conductivity) to a sum of reciprocal path lengths. ${ }^{20}$ These considerations are not restricted to perfect crystals or the solid phase, as even highly structured liquids can exhibit nanoscale ballistic effects with mean free paths lengths of several nanometers. ${ }^{24}$

It is increasingly understood that the structure of shocked explosives in bulk regions and near hot spots is far from that of a perfect compressed crystal. Many organic molecular explosives exhibit plastic failure mechanisms that generate a nanoscale network of amorphous-like regions, colloquially termed "shear bands". ${ }^{27-31}$ Regions near a hot spot core are even more strongly sheared ${ }^{32}$ and can also emit shear bands. ${ }^{7,33}$ These sheared regions exhibit both high temperatures and mechanochemically accelerated reaction kinetics. ${ }^{31,32,34}$ Figure 1 shows an all-atom prediction for the structure of a typical hot spot in TATB, which clearly exhibits a range of sheared states that are interspersed by crystalline regions. The combined effect of plastically deformed regions and their boundaries may reduce the apparent conductivity considerably from values for the perfect crystal. It it not well understood if continuum theories 


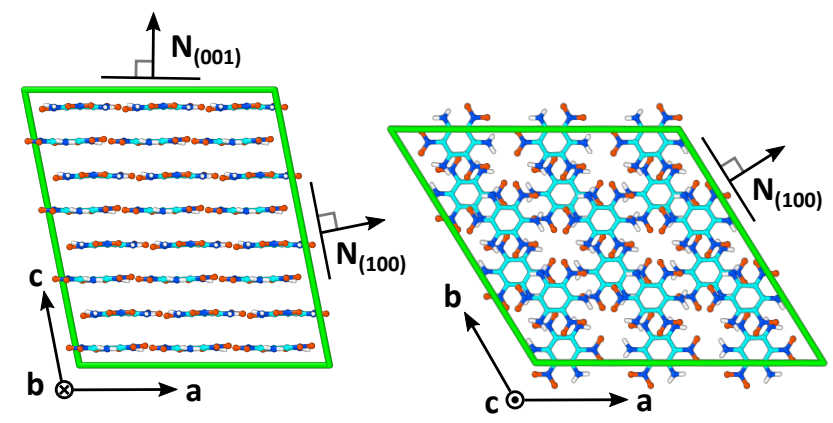

Figure 2: Supercell representation of TATB crystal structure rendered using OVITO ${ }^{25}$ with atoms colored cyan, blue, red, and white for carbon, nitrogen, oxygen, and hydrogen. The two shock directions considered in this work are aligned with the (100) and (001) crystal face normal vectors $\mathbf{N}_{(100)}$ and $\mathbf{N}_{(001)}$.

for heat transport, such as Fourier's heat law and the diffusive heat equation (HEq), hold for nanoscale explosive hot spots. A related consideration is to determine what comprises a "reasonable" effective thermal conductivity for heat transport on the nanoscale in explosives under shock initiation or detonation regimes.

This work focuses on the thermal response of hot spots in TATB, which is a layered molecular crystal that exhibits some of the largest and most anisotropic thermal conductivity values for an explosive. The crystal is comprised of planar molecular layers that stack in the c lattice direction (see Figure 2). ${ }^{35}$ Molecules within a layer form an extensive 2D hydrogen bonding network, while molecules in adjacent layers interact primarily by weak van der Waals forces. Large and anisotropic conductivity values are typical of many hydrogenbonded materials. ${ }^{15,24,36,37}$ In these respects, TATB provides an excellent bounding case for thermal physics of explosives, and for anisotropy in hydrogen-bonded materials more generally. A variety of orientation-dependent deformation mechanisms can activate in bulk TATB single crystal under shock and non-shock loads, including layer sliding, ${ }^{16,38-41}$ buckling/twinning, ${ }^{40,42,43}$ and dislocations that break the planar crystal layers ${ }^{40}$ whose hindered motions may serve as nuclei for shear band growth. ${ }^{43}$ The anisotropic thermal conductivity of TATB has been extensively characterized through molecular dynamics (MD) simulations as a function of temperature and pressure for both perfect (and near perfect) single crys- 
tals $^{15-19}$ as well as the liquid state. ${ }^{44}$ Near room temperature and pressure, the conductivity along directions within the molecular layers along $\mathbf{N}_{(100)}$ is $\approx 64 \%$ greater than the conductivity for the direction normal to the layers along $\mathbf{N}_{(001)}$. Modeling estimates for the average phonon mean free path length in perfect TATB crystal are on the order of a few nanometers or less, ${ }^{45}$ which suggests that thermal relaxation of nanoscale hot spots may be primarily diffusive.

We employ a combination of non-reactive all-atom MD modeling and HEq modeling to explore the thermal relaxation characteristics of realistic hot spots in TATB explosive. Nonreactive MD simulations provide direct "full-physics" predictions for the formation ${ }^{32,46-49}$ and relaxation ${ }^{32}$ of hot spots that implicitly includes all mechanical heat generation mechanisms and ballistic transport effects within the classical approximation for phonon populations and the heat capacity. The application of a non-reactive MD model allows for isolating thermal transport without the added complication of chemical reactions leading to heat generation. ${ }^{50-52}$ Initial hot spot structures are sampled through simulations of pore collapse in TATB single crystals with two carefully chosen orientations that provide upper and lower bounds for thermal conductivity anisotropy relative to normal conditions. A recently developed computational approach, ${ }^{32}$ shock trapping internal boundaries (STIBs), is used to drastically extend the MD simulation time to obtain relaxation characteristics of hot spots out to nanosecond timescales. Applicability of Fourier's heat law is assessed by fitting HEq models to the MD predictions in which the thermal conductivity is treated as an adjustable parameter. The results suggest that diffusive HEq models provide a surprisingly accurate representation of thermal physics of nanoscale explosive hot spots, even with a number of simplifications such as neglecting anisotropy, explicit functional dependence on temperature or local phase/disorder, and additional mechanical sources for heat generation. 

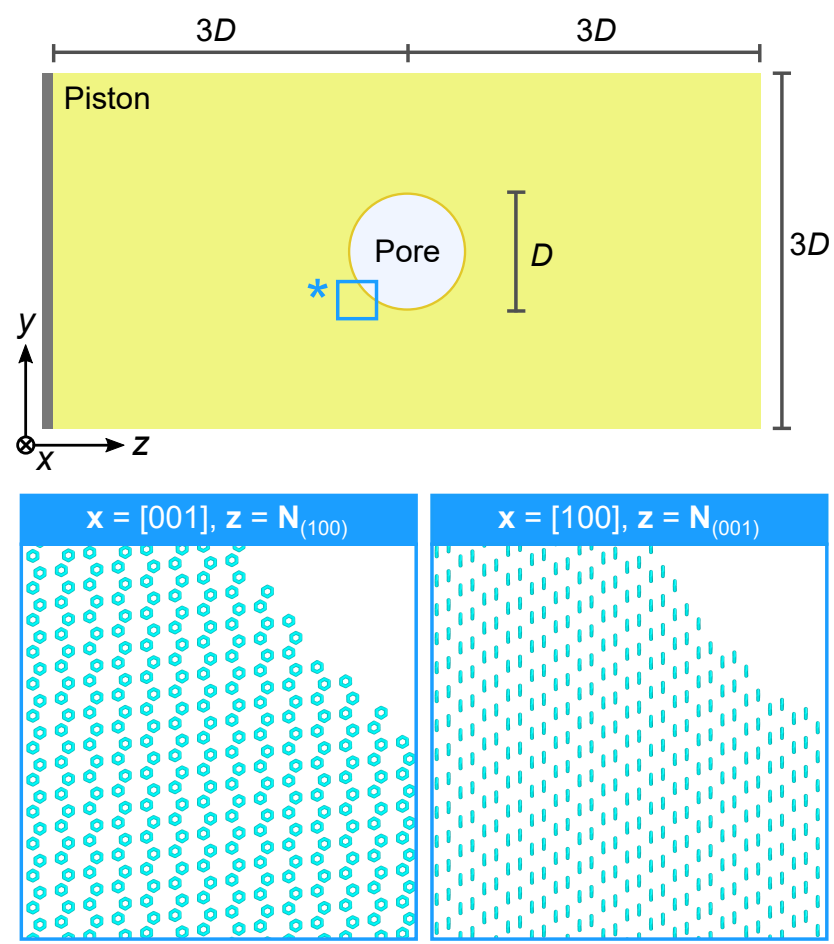

Figure 3: Schematic for initial condition in reverse-ballistic shock simulations of pore collapse. The sample is impacted on the piston, generating a shock that travels in the $+z$ direction. Crystal packing near the pore is shown for the two shock directions considered, with snapshots of the TATB $\mathrm{C}_{6}$ rings rendered using OVITO. ${ }^{25}$

\section{Methods}

\subsection{Molecular Dynamics Simulations}

Classical all-atom molecular dynamics (MD) simulations were performed using LAMMPS, ${ }^{53}$ 3Dperiodic simulation cells, and a variant of the well-established non-reactive force field (FF) for TATB developed by Bedrov et al. ${ }^{54}$ The variant used here incorporates tuned harmonic bond and angle potentials ${ }^{15}$ and a repulsive intramolecular potential between $\mathrm{O}$ and $\mathrm{H}$ atoms. ${ }^{55}$ All $\mathrm{N}-\mathrm{H}$ bonds were constrained to their equilibrium values using RATTLE $^{56,57}$ with the relative accuracy threshold set to $10^{-4}$. Non-bonded terms were evaluated in real space up to an $11 \AA$ cutoff and the electrostatic terms were evaluated using the Wolf potential ${ }^{58}$ with an $11 \AA$ cutoff.

Dynamically formed hot spots were generated through reverse-ballistic direct shock sim- 
ulations $^{59}$ of pore collapse in oriented TATB crystals. A schematic of the pre-shock configuration is shown in Figure 3. With a reverse-ballistic configuration, a material sample is impacted on a rigid piston (or momentum mirror) with velocity $\mathbf{U}_{\mathrm{p}}$, which produces a shock with velocity $\mathbf{U}_{\mathrm{w}}$ with respect to the stationary piston and $\mathbf{U}_{\mathrm{s}}=\mathbf{U}_{\mathrm{w}}-\mathbf{U}_{\mathrm{p}}$ with respect to the unshocked material. Simulations were performed using a quasi-2D geometry in which the $y$ and $z$ dimensions were extended and the $x$ dimension was thin (approximate $x y z$ dimensions are $4 \mathrm{~nm} \times 120 \mathrm{~nm} \times 270 \mathrm{~nm})$. An initial cylindrical pore of vacuum space with diameter $D=40 \mathrm{~nm}$ and axis along $x$ was formed in the geometric center of a cell that had approximate $y-z$ dimensions $3 D \times 6 D$. A $5 \mathrm{~nm}$ vacuum space was added along $z$ to lift the periodicity in that direction and prevent self-interactions. Prior to shock generation, each configuration was equilibrated through a $25 \mathrm{ps}$ isochoric-isothermal (NVT) simulation performed using a Nosé-Hoover-style thermostat ${ }^{60,61}$ with the coupling parameter set to 100 fs and a 0.5 fs time step. Shock trajectories were integrated using isochoric-isoenergetic $(N V E)$ equations of motion and a 0.2 fs time step.

Two shock directions were considered, with one being normal to the (100) crystal face along $\mathbf{N}_{(100)}=\mathbf{b} \times \mathbf{c}$ and the other normal to the (001) crystal face along $\mathbf{N}_{(001)}=\mathbf{a} \times \mathbf{b}$. These two directions are respectively approximately within and exactly normal to the TATB crystal layers and are oriented along $\mathbf{z}$ in the lab frame. The cylinder axes were chosen to align with specific lattice directions. For the $\mathbf{N}_{(100)}$-oriented cell, the cylinder axis was along [001] (lattice vector $\mathbf{c}$ ), and for the $\mathbf{N}_{(001)}$-oriented cell, the cylinder axis was along [100] (lattice vector a). The Generalized Crystal Cutting Method ${ }^{62}$ (GCCM) was used to construct both cells starting from primitive cell lattice parameters determined with the TATB FF at $300 \mathrm{~K}$ and 1 atm. ${ }^{55}$ These two shock directions yield upper and lower bounds on the anisotropy of many physical properties in the $y$ - $z$ plane, including elastic mechanical compliance ${ }^{54}$ and thermal conductivity. ${ }^{15}$

Four impact speeds were considered for each direction, namely $\left|\mathbf{U}_{\mathrm{p}}\right|=0.5,1.0,1.5$, and $2.0 \mathrm{~km} \cdot \mathrm{s}^{-1}$, which correspond to shock stresses of $\sigma_{z z}=3 \pm 1,8 \pm 1,15 \pm 2$, and $22 \pm 2 \mathrm{GPa}$. We 
focus here on predicting and understanding the long timescale thermal relaxation of hot spots that are formed from pore collapse. In direct shock simulations, the "end" of the simulation occurs when the shock wave reaches the far free surface. We extend the timescale beyond this point for the material region near the hot spot using shock trapping internal boundaries (STIBs), ${ }^{32}$ which are a generalization of shock absorbing boundary conditions. ${ }^{27,63}$ In this approach (see Figure 4), two STIB regions are defined in which molecules are held in rigid positions once the simulation reaches maximum compression when the primary wave reaches the end of the cell. The hot spot region between the STIBs is thus isolated from the various primary and reflected waves in the system. The dynamics of the flexible hot spot region were propagated using $N V E$ equations of motion subject to the approximately uniform external field generated by explicitly computed interactions with the rigid STIB molecules. The STIB regions were $3 \mathrm{~nm}$ thick, which is more than twice the cutoffs used in the TATB FF, and the material outside the STIBs was deleted to improve computational efficiency. Initial shock simulations to generate hot spots had $\approx 12$ million atoms and the relaxation simulations with STIBs had $\approx 5$ million atoms.

Temperature fields were obtained from the MD simulations through a multistep analysis. Starting from the atomic velocities $\mathbf{v}_{i}$, we computed the total molecular kinetic energy $K_{\text {tot }}$ as

$$
K_{\mathrm{tot}}=\sum \frac{1}{2} m_{i} \mathbf{v}_{i} \cdot \mathbf{v}_{i}
$$

where $m_{i}$ is the mass of atom $i$ and the sum runs over all 24 atoms in the molecule. Given that the post-shock material is at rest in the lab frame, this provides a measure of molecular temperature when converted to Kelvin units through

$$
T=\frac{2 K_{\mathrm{tot}}}{66 k_{\mathrm{B}}}
$$

Here, the factor of 66 arises from the non-constrained degrees of freedom in a TATB molecule. These molecular temperature values were then averaged within a sphere of radius $1.5 \mathrm{~nm}$ 


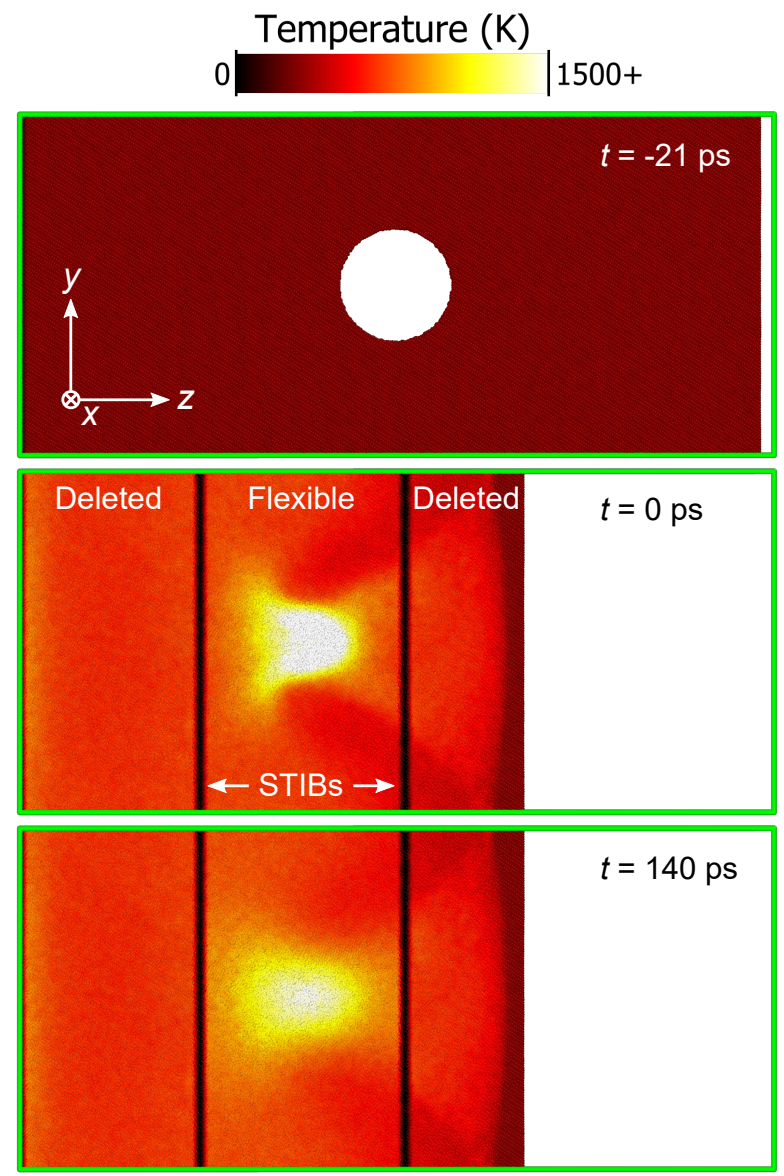

Figure 4: Snapshots showing the application of STIBs to study hot spot relaxation. The STIBs are applied at maximum compression following shock-induced pore collapse, which is taken to the be the time origin $t=0 \mathrm{ps}$ in this study. Images were rendered using OVITO ${ }^{25}$ and show molecular temperature with periodic boundaries indicated by green lines.

about each molecule to smooth local fluctuations. Finally, we obtained the temperature profile $T_{\mathrm{MD}}(y, z, t)$ on a grid through 2D Eulerian binning based on molecular center of mass positions using $\Delta y=1.003 \mathrm{~nm}$ and $\Delta z=0.952 \mathrm{~nm}$ for the $\mathbf{N}_{(100)}$ direction cell and $\Delta y=1.019 \mathrm{~nm}$ and $\Delta z=0.993 \mathrm{~nm}$ for the $\mathbf{N}_{(001)}$ direction cell. (These $\Delta y$ and $\Delta z$ values arose from dividing the primary simulation cell into $120 \times 280$ bins.)

\subsection{Heat Equation Model}

Molecular dynamics predictions of hot spot relaxation were fit to transient solutions of the 2D diffusive heat equation ( $\mathrm{HEq}$ ) treating the anisotropic thermal conductivity $\lambda$ as an 
adjustable parameter. This approach closely follows earlier works that fit MD predictions to 1D HEq models. ${ }^{45,64}$ Neglecting off-diagional components of the conductivity tensor, the HEq becomes

$$
\frac{\partial T(y, z, t)}{\partial t}=\frac{\lambda_{y}}{C_{V}} \frac{\partial^{2} T(y, z, t)}{\partial y^{2}}+\frac{\lambda_{z}}{C_{V}} \frac{\partial^{2} T(y, z, t)}{\partial z^{2}} .
$$

Here, the thermal conductivity (units $\mathrm{W} \cdot \mathrm{m}^{-1} \cdot \mathrm{K}^{-1}$ ) is separated into components for the $y$ and $z$ directions and $C_{V}$ is the volumetric heat capacity (units $\mathrm{J} \cdot \mathrm{m}^{-3} \cdot \mathrm{K}^{-1}$ ). We obtained values for $C_{V}$ based on the classical harmonic molecular specific heat $\left(66 k_{\mathrm{B}}\right.$ with constrained $\mathrm{N}-\mathrm{H}$ vibrations) and the average post-shock density $\rho$ for each simulation. Density variations within a given simulation domain were not considered in this approach.

Solutions to Eq. 3 were obtained numerically on a grid using an Euler-type propagator ${ }^{65}$

$$
T_{i, j}^{n+1}=T_{i, j}^{n}+\frac{\lambda_{y} \Delta t}{C_{V}} \cdot \frac{T_{i+1, j}^{n}-2 T_{i, j}^{n}+T_{i-1, j}^{n}}{\Delta y^{2}}+\frac{\lambda_{z} \Delta t}{C_{V}} \cdot \frac{T_{i, j+1}^{n}-2 T_{i, j}^{n}+T_{i, j-1}^{n}}{\Delta z^{2}}
$$

where the $y$ dimension is indexed by $i$, the $z$ dimension is indexed by $j$, and time is indexed by $n$. The time step $\Delta t$ was set to $100 \mathrm{fs}$ and the $\Delta y$ and $\Delta z$ grid spacing values were chosen to match those from the grid-based analysis of the temperature field in the MD simulations ( $\Delta y \approx \Delta z \approx 1 \mathrm{~nm}$ ). The boundary condition for the $y$ direction was treated as periodic to match the MD simulations, and the condition for $z$ was treated as adiabatic. An adiabatic boundary is reasonable as the rigid molecules in the STIB regions cannot exchange energy with the flexible region. Shearing of the flexible molecules against rigid STIB molecules is a potential source for heat within the flexible region, but this is neglected in the present treatment.

Solutions to the HEq were propagated in time starting from initial temperature fields determined from the MD simulations. The time origin was taken to be when the STIB regions were imposed in the MD simulatons. Optimum values for $\lambda_{y}$ and $\lambda_{z}$ were determined 
through a parametric sweep as the values which minimize the root mean-square error

$$
\operatorname{RMSE}=\left\{\frac{1}{N_{y} N_{z} N_{t}} \sum_{i, j, n}^{N_{y}, N_{z}, N_{t}}\left[T_{\mathrm{MD}}\left(y_{i}, z_{j}, t_{n}\right)-T_{\mathrm{HEq}}\left(y_{i}, z_{j}, t_{n} ; \lambda_{y}, \lambda_{z}\right)\right]^{2}\right\}^{1 / 2},
$$

between the MD-predicted time-dependent temperature field $T_{\mathrm{MD}}\left(y_{i}, z_{j}, t_{n}\right)$ and that obtained from the HEq, denoted $T_{\mathrm{HEq}}\left(y_{i}, z_{j}, t_{n} ; \lambda_{y}, \lambda_{z}\right)$.

There are three assumptions that enter the HEq model in its present application. The first is that we treat the conductivity as a constant with no off-diagonal $\left(\lambda_{y z}\right)$ component. While not strictly zero, those terms are much smaller than the diagonal components for TATB. ${ }^{18}$ Second, we neglect heat generation terms that could arise internally or at the boundaries in $z$. This is not unreasonable as mechanical work is the only potential heat source in our non-reactive simulations and the system is largely stationary by the time the STIBs are imposed. The last is that we treat the heat capacity as a constant using the classical expression, which best matches the physics in our classical MD simulations.

\section{$3 \quad$ Results and Discussion}

\subsection{Hot Spot Relaxation Characteristics}

Realistic initial hot spot temperature fields for oriented TATB single crystals were sampled through direct shock simulations at four different impact speeds $\left|\mathbf{U}_{\mathrm{p}}\right|$. Two shock directions were chosen that minimize $\left(\mathbf{N}_{(100)}\right)$ and maximimze $\left(\mathbf{N}_{(001)}\right)$ the initial relative anisotropy of the thermal conductivity in the $y$ and $z$ directions. In the former case, both the $y$ and $z$ directions are within the plane of the crystal layers, which has a predicted conductivity of $\lambda=0.95 \mathrm{~W} \cdot \mathrm{m}^{-1} \cdot \mathrm{K}^{-1}$ at room temperature and pressure. ${ }^{17}$ In the latter case, the $y$ direction is within the plane of the layers and $z$ points between the layers, which has a predicted conductivity of $\lambda=0.58 \mathrm{~W} \cdot \mathrm{m}^{-1} \cdot \mathrm{K}^{-1}$, so $\lambda_{y}$ and $\lambda_{z}$ initially differ by $64 \% .{ }^{17}$

Figure 5 shows the initial hot spot temperature fields generated for the $\mathbf{N}_{(100)}$ shock 

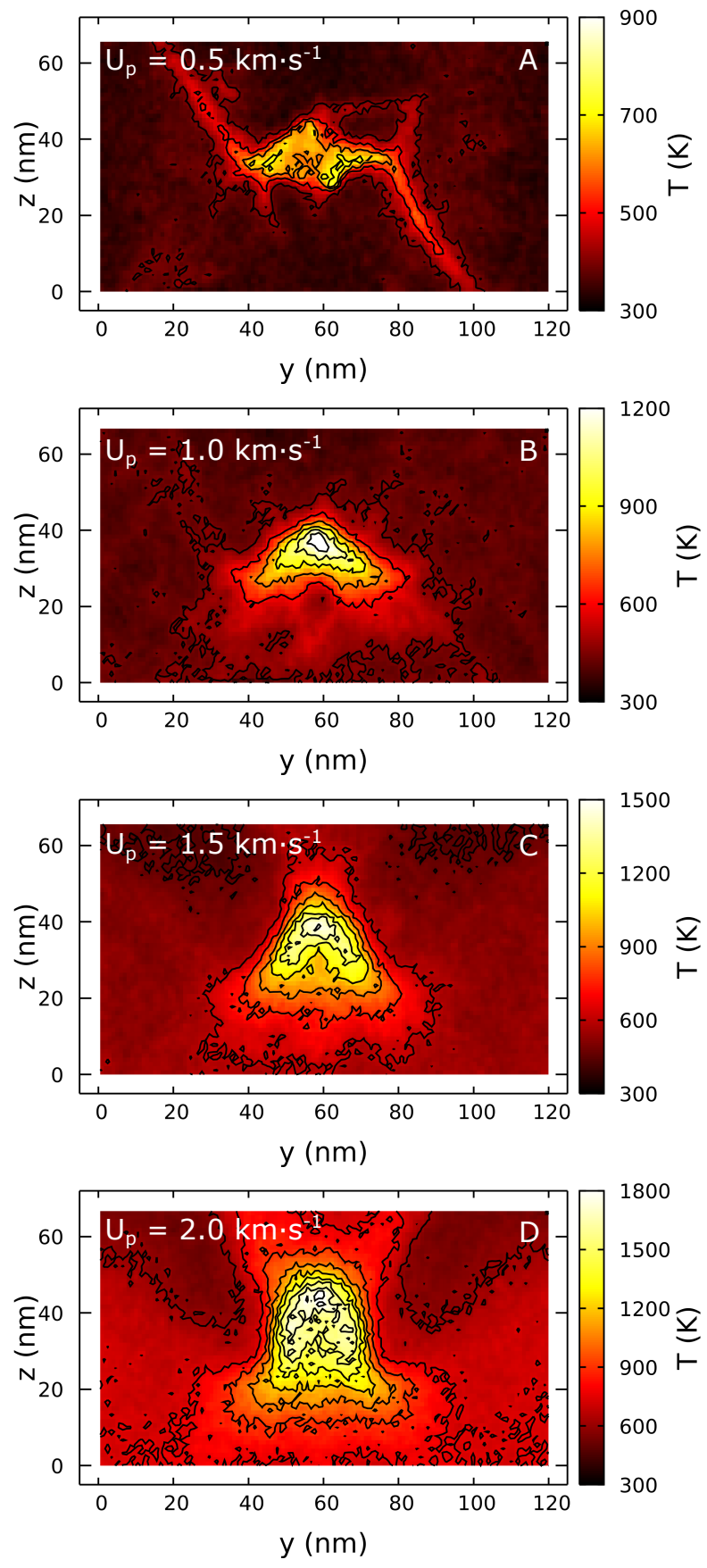

Figure 5: Initial hot spot temperature fields generated at collapsed pores in shocked $\mathbf{N}_{(100)^{-}}$ oriented TATB crystal. The piston velocity and bulk shock pressure for the cases are: (A) $\left|\mathbf{U}_{\mathrm{p}}\right|=0.5 \mathrm{~km} \cdot \mathrm{s}^{-1}, \sigma_{z z}=3 \mathrm{GPa} ;(\mathrm{B})\left|\mathbf{U}_{\mathrm{p}}\right|=1.0 \mathrm{~km} \cdot \mathrm{s}^{-1}, \sigma_{z z}=8 \mathrm{GPa} ;(\mathrm{C})\left|\mathbf{U}_{\mathrm{p}}\right|=1.5 \mathrm{~km} \cdot \mathrm{s}^{-1}$, $\sigma_{z z}=15 \mathrm{GPa}$; and (D) $\left|\mathrm{U}_{\mathrm{p}}\right|=2.0 \mathrm{~km} \cdot \mathrm{s}^{-1}, \sigma_{z z}=22 \mathrm{GPa}$. 


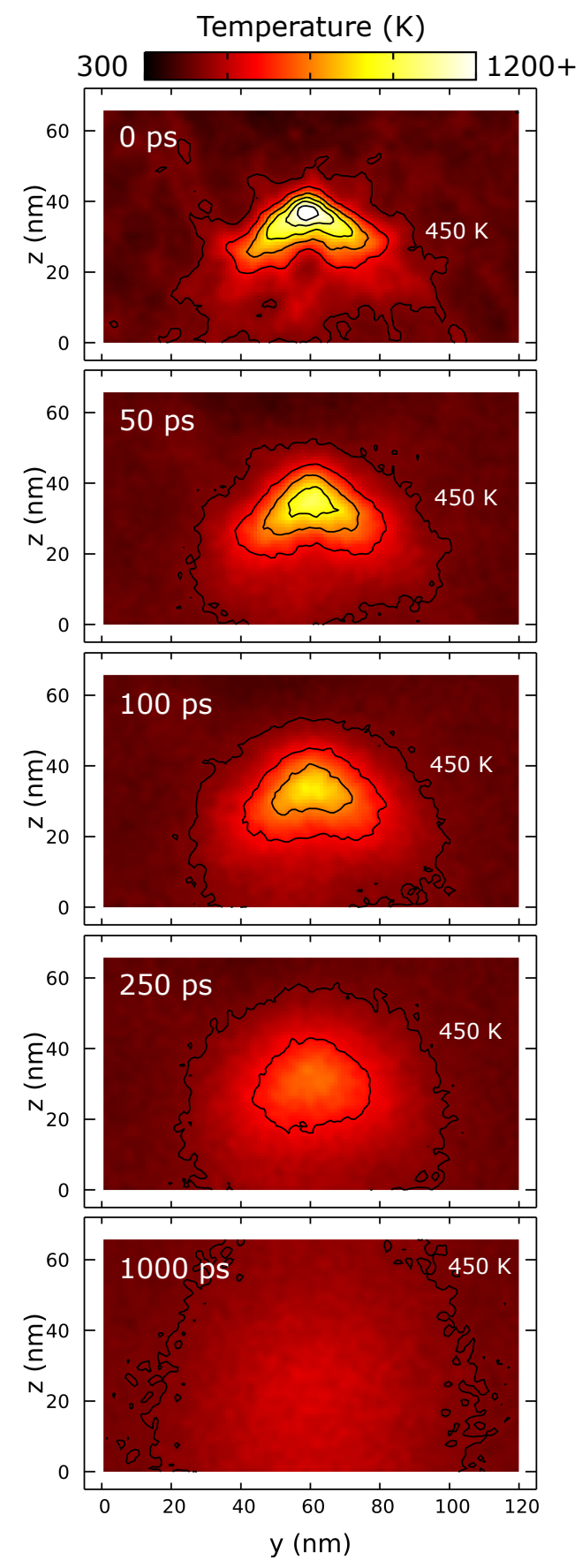

Figure 6: Molecular dynamics predictions for the non-reactive evolution of the hot spot temperature field for the case with $\mathbf{N}_{(100)}$ and $\left|\mathbf{U}_{\mathrm{p}}\right|=1.0 \mathrm{~km} \cdot \mathrm{s}^{-1}$. Snapshots show the temperature field at selected times up to the end of the MD simulations (1000 ps) with contours drawn in $150 \mathrm{~K}$ intervals starting at $450 \mathrm{~K}$. 

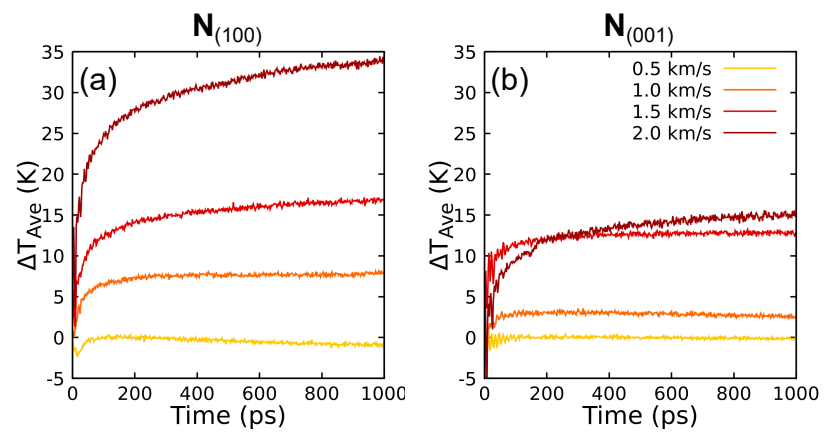

Figure 7: Change in the system average temperature during MD simulations of hot spot relaxation for (a) the $\mathbf{N}_{(100)}$ shock direction case and (b) the $\mathbf{N}_{(001)}$ shock direction case.

direction at the four different shock strengths considered. It is evident that shock strength influences both the maximum temperature and shape of the hot spot. Differences in shape arise due to a transition in the pore collapse mechanism, from a highly viscosplastic process for weak shocks to a hydrodynamic jetting response for strong shocks. In the weakest shock case, the hot spot is more delocalized due to dynamically formed shear bands that radiate from the core of the hot spot. The background temperature field of the bulk crystal is hotter in the stronger shock cases, as expected from differences in work due to volumetric compression. While not shown, the initial hot spots for the $\mathbf{N}_{(001)}$ case exhibit similar trends with increasing shock strength.

Long timescale relaxation responses were predicted through 1000 ps long MD simulations in which STIBs were employed to isolate the hot spot regions. Snapshots of the temperature field at various points in time are shown in Figure 6 for an example case with shock direction $\mathbf{N}_{(100)}$ and piston velocity $\left|\mathbf{U}_{\mathrm{p}}\right|=1.0 \mathrm{~km} \cdot \mathrm{s}^{-1}$. The initial hot spot temperature field at $t=0 \mathrm{ps}$ has a core temperature slightly in excess of $1200 \mathrm{~K}$. Comparison of the panels in Figure 6 shows a steady reduction in core temperature as the hot spot dissipates due to thermal conduction. The peak temperature decreases by roughly $300 \mathrm{~K}$ in $100 \mathrm{ps}$, and the hot spot is nearly completely dissipated by 1000 ps.

As the hot spot region evolves according to $N V E$ equations of motion in an external field generated by the STIB molecules, the dynamics are not rigorously conservative. How- 
ever, we find that the total energy is constant to within $0.002 \mathrm{kcal} \cdot \mathrm{mol}^{-1} \cdot \mathrm{molecule}^{-1}$. The qualitative features of the time-dependent temperature field show little indication for any additional mechanical heat generation. Total system temperature provides a scalar metric for heat sources arising from interactions of flexible molecules with the rigid STIB regions and/or from mechanical relaxation of strained states within the hot spot region that release latent potential energy as heat. ${ }^{32}$ Inspection of the change in total system temperature in Figure 7 (a) reveals a modest mechanical relaxation in the strongest shock case for the $\mathbf{N}_{(100)}$ direction that increases the average temperature by $34 \mathrm{~K}$, from $787.1 \mathrm{~K}$ to $821.1 \mathrm{~K}$. This amounts to a $4.1 \%$ error relative to the final temperature. The next strongest shock with $\left|\mathbf{U}_{\mathrm{p}}\right|=1.5 \mathrm{~km} \cdot \mathrm{s}^{-1}$ has a total system temperature increase of $17 \mathrm{~K}$, which amounts to a $2.8 \%$

error. Analogous results for the $\mathbf{N}_{(001)}$ shock direction in Figure $7(\mathrm{~b})$ indicate a lower overall amount of mechanical relaxation for a given shock strength in that case. While additional heat generation is not strictly zero, accounting for it in a spatial sense is nontrivial and the overall magnitude is small enough to justify neglecting heat source terms in Equation 3.

\subsection{Fitted Heat Equation Models}

Anisotropic heat equation models were fit to the MD predictions to obtain optimum thermal conductivity values that minimize the RMSE error in the temperature field. In all cases, the HEq model was initialized using the MD-predicted temperature profile $T_{\mathrm{MD}}\left(y_{i}, z_{j}, t_{n}\right)$ at time $t=0 \mathrm{ps}$, which is taken to be when the STIBs were imposed. Subsequent measurements of $T_{\mathrm{MD}}\left(y_{i}, z_{j}, t_{n}\right)$ at later times serve only to constrain the HEq model fitting. A parametric scan of conductivity values was performed over the interval $0.05 \mathrm{~W} \cdot \mathrm{m}^{-1} \cdot \mathrm{K}^{-1} \leq \lambda_{y}, \lambda_{z} \leq$ $3.00 \mathrm{~W} \cdot \mathrm{m}^{-1} \cdot \mathrm{K}^{-1}$ in increments of $0.05 \mathrm{~W} \cdot \mathrm{m}^{-1} \cdot \mathrm{K}^{-1}$ to identify those values yielding the lowest RMSE error (Eq. 5). Each relaxation simulation was $1000 \mathrm{ps}$ long and $T_{\mathrm{MD}}\left(y_{i}, z_{j}, t_{n}\right)$ was sampled every 2 ps. Parameters for these fits are collected in Table 1 and plots of the resulting RMSE surfaces are shown in Figure 8.

A number of features are apparent from inspection of Table 1 and the RMSE surfaces 


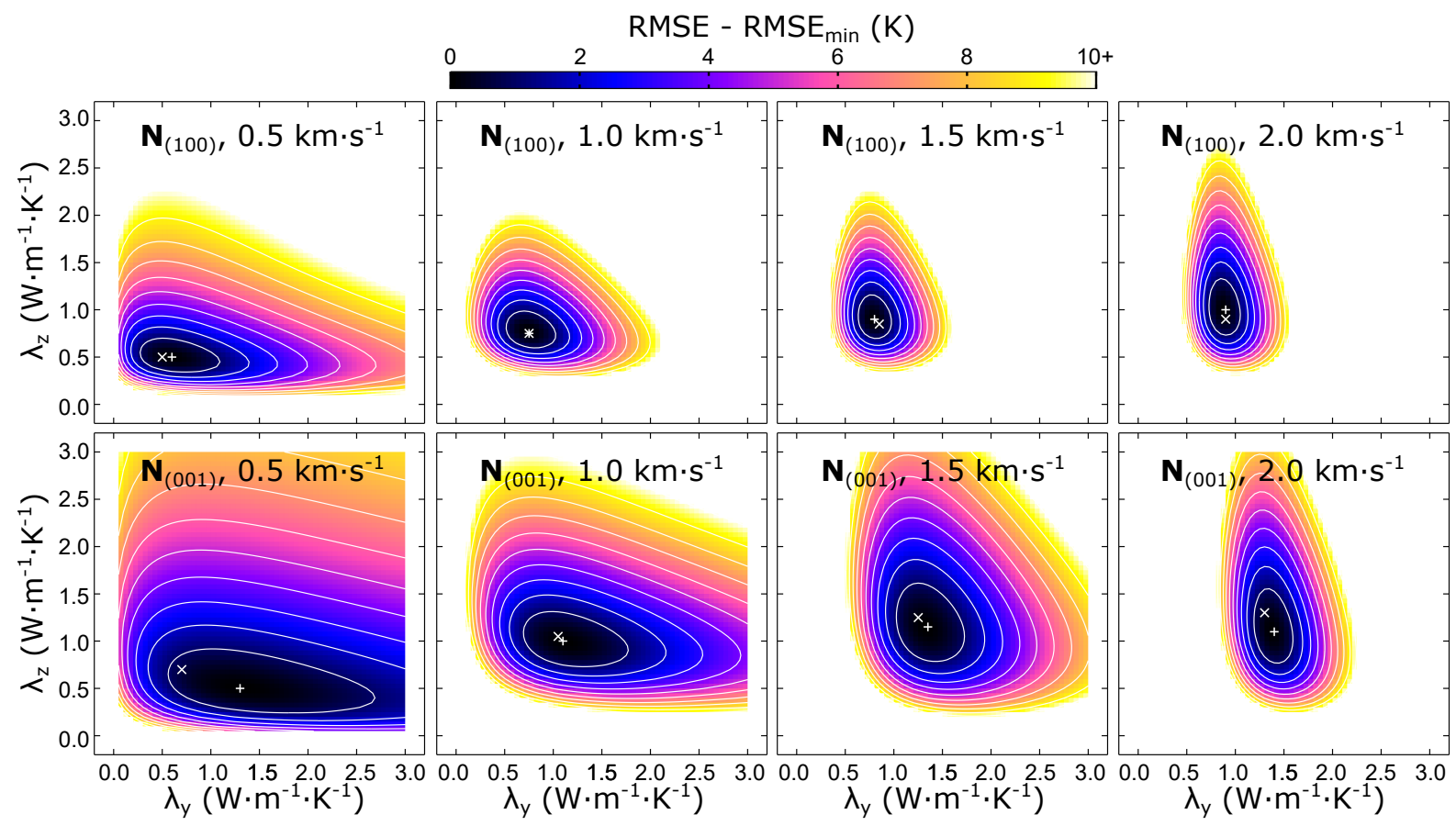

Figure 8: Root mean-square error surfaces for fitting MD predictions for hot spot relaxation to heat equation models by varying the thermal conductivity. Each surface measures the RMSE (Eq. 8) relative to the minimum values, which are reported in Table 1 . The optimum anisotropic conductivity is denoted by "+" and the optimum isotropic conductivity $\lambda_{\text {iso }}$ is denoted by " $\times$ " on each surface. Contours are drawn in $1 \mathrm{~K}$ increments. 
Table 1: Optimized thermal conductivity values from fitting hot spot relaxation.

\begin{tabular}{cccccccc}
\hline \hline $\begin{array}{c}\text { Shock } \\
\text { Direction }\end{array}$ & $\begin{array}{c}\left|\mathrm{U}_{\mathrm{p}}\right| \\
\left(\mathrm{km} \cdot \mathrm{s}^{-1}\right)\end{array}$ & $\begin{array}{c}\rho \\
\left(\mathrm{g} \cdot \mathrm{cm}^{-3}\right)\end{array}$ & $\begin{array}{c}\lambda_{y}{ }^{a} \\
\left(\mathrm{~W} \cdot \mathrm{m}^{-1} \cdot \mathrm{K}^{-1}\right)\end{array}$ & $\begin{array}{c}\lambda_{z}{ }^{b} \\
\left(\mathrm{~W} \cdot \mathrm{m}^{-1} \cdot \mathrm{K}^{-1}\right)\end{array}$ & $\begin{array}{c}\lambda_{\text {iso }} \\
\left(\mathrm{W} \cdot \mathrm{m}^{-1} \cdot \mathrm{K}^{-1}\right)\end{array}$ & $\begin{array}{c}\mathrm{RMSE}_{\min } \\
(\mathrm{K})\end{array}$ & $\begin{array}{c}\text { Rel. RMSE } \\
\text { Rin } \\
(\%)\end{array}$ \\
\hline $\mathbf{N}_{(100)}$ & 0.5 & 1.481 & 0.60 & 0.50 & 0.50 & 8.8 & 5.0 \\
& 1.0 & 1.678 & 0.75 & 0.75 & 0.75 & 12.5 & 4.7 \\
& 1.5 & 1.824 & 0.80 & 0.90 & 0.85 & 20.0 & 5.0 \\
& 2.0 & 1.962 & 0.90 & 1.00 & 0.90 & 38.0 & 5.5 \\
\hline $\mathbf{N}_{(001)}$ & 0.5 & 2.082 & 1.30 & 0.50 & 0.70 & 10.2 & 5.5 \\
& 1.0 & 2.355 & 1.10 & 1.00 & 1.05 & 10.5 & 4.6 \\
& 1.5 & 2.549 & 1.35 & 1.15 & 1.25 & 19.8 & 5.3 \\
& 2.0 & 2.735 & 1.40 & 1.10 & 1.30 & 25.4 & 4.9 \\
\hline \hline
\end{tabular}

$a \overline{\bar{T}} \overline{\text { The } y \text { direction is aligned with [052] for the } \mathbf{N}_{(100)} \text { shock direction case and with [120] for }}$ the $\mathbf{N}_{(001)}$ shock direction case.

${ }^{b}$ The $z$ direction coincides with the shock direction in both cases.

in Figure 8. The post-shock densities span a wide range relative to the density of perfect crystal at room temperature and pressure $\left(\rho_{\mathrm{cr}}=1.93 \mathrm{~g} \cdot \mathrm{cm}^{-3}\right)$, and are highly dependent on the shock direction and strength. Values lower than $\rho_{\text {cr }}$ are not unexpected as the pre-shock configuration was far from theoretical maximum density due to inclusion of a pore. It is also expected that the density increases with shock strength. It is perhaps interesting that the highest density reached for shocks along $\mathbf{N}_{(100)}$ is lower than the lowest value for shocks along $\mathbf{N}_{(001)}$. These differences may arise due to an order of magnitude difference in elastic stiffness of the crystal along those two directions. The crystal is much more compressible normal to the layers along $\mathbf{N}_{(001)}$ than it is within the layers along $\mathbf{N}_{(100)}$, with elastic coefficients $C_{11}=3.4 C_{33}$ near normal conditions. ${ }^{54}$

The optimum anisotropic conductivity values obtained for the two shock directions yield similar trends with shock strength. Both systems exhibit an overall increase in the conductivity with increasing shock strength and density. Despite situations with apparent anisotropy, the RMSE surfaces are slowly varying about their respective minimums. The local curvature in the RMSE surfaces generally increases with increasing shock strength. In all instances, an isotropic conductivity model ( $\times$ symbols) yields an RMSE that is within $1 \mathrm{~K}$ of the minimum obtained with an anisotropic model ( + symbols). This is true even for the case with the most pronounced anisotropy $\left(\mathbf{N}_{(001)},\left|\mathbf{U}_{\mathrm{p}}\right|=0.5 \mathrm{~km} \cdot \mathrm{s}^{-1}\right)$. Relative RMSE values computed with respect to the system-average temperature at $t=1000 \mathrm{ps}$ are approximately $5 \%$ and do 
not show a significant trend with shock direction or strength. As TATB is perhaps the most anisotropic organic molecular crystalline explosive, anisotropy in thermal transport may be a broadly negligible effect at shock initiation conditions for most explosives.

\subsection{Inspection of Isotropic Fits}

Detailed comparisons of isotropic fitted HEq models for the $\mathbf{N}_{(100)}$ and $\mathbf{N}_{(001)}$ shock directions at the largest shock strength are resepctively shown in Figures 9 and 10. Heat maps of the temperature profile from $\mathrm{MD}$ and the $\mathrm{HEq}$ model $\left(T_{\mathrm{MD}}\right.$ and $\left.T_{\mathrm{HEq}}\right)$ are shown at early and late times, along with the difference between the two $T_{\mathrm{MD}}-T_{\mathrm{HEq}}$. This last quantity is the signed error contribution that enters into Eq. 5. In each case, the HEq model is fit to the entire time series with equal weight, so both early and late-time discrepancies are simultaneously minimized.

Focusing first on the MD predictions (left column), the initial hot spot core in the $\mathbf{N}_{(100)}$ case is more centralized and elliptical compared to the horse-shoe shaped hot spot in the $\mathbf{N}_{(001)}$ case. The latter reaches a higher initial maximum temperature in a very small region. After $250 \mathrm{ps}$, the $\mathbf{N}_{(001)}$ hot spot core relaxes to an approximate elliptical symmetry that is similar in size and temperature to the $\mathbf{N}_{(100)}$ one.

Qualitative and even significant quantitative agreement is evident between MD predictions and the HEq models for both shock directions. Visual comparison of $T_{\mathrm{MD}}$ and $T_{\mathrm{HEq}}$ shows that the HEq captures the qualitative shape and temperature magnitude of both hot spots at all times. A more quantitative assessment is obtained from spatially mapping the error (left column). Both cases exhibit similar errors at early and late times. At 50 ps, this is characterized by the HEq under predicting the MD temperature in the hot spot core $\left(T_{\mathrm{MD}}-T_{\mathrm{HEq}}>0\right)$ while it over predicts just outside the core $\left(T_{\mathrm{MD}}-T_{\mathrm{HEq}}<0\right)$. The magnitude of the difference in any particular region is much lower at $1000 \mathrm{ps}$.

The late time signed error is almost entirely positive in the $\mathbf{N}_{(100)}$ case. This is due to the more significant mechanical relaxation in that case, which leads to a $34 \mathrm{~K}$ rise in the 


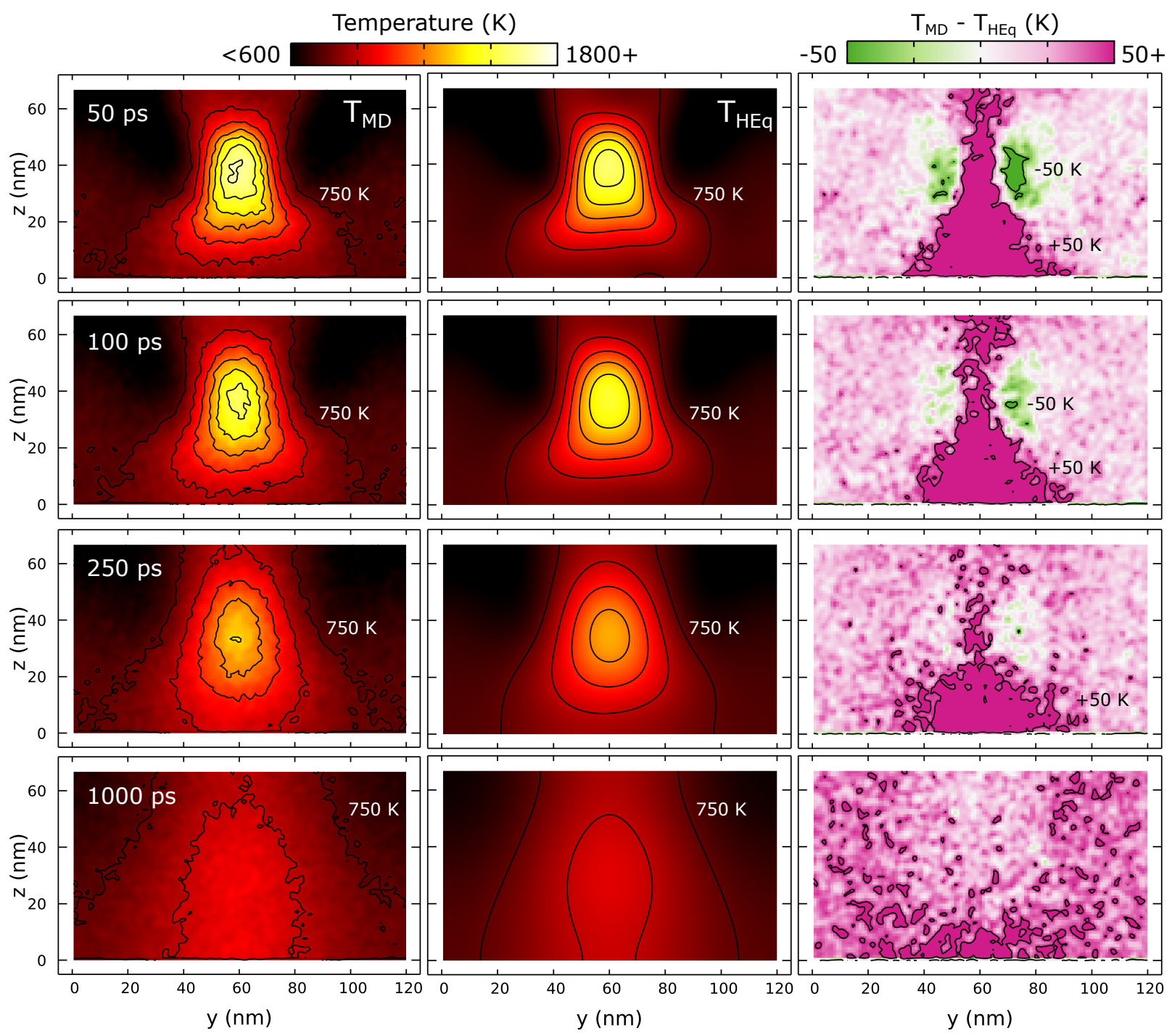

Figure 9: Comparison of temperature profiles from MD and an isotropic fitted HEq model for the case with $\mathbf{N}_{(100)}$ and $\left|\mathbf{U}_{\mathrm{p}}\right|=2.0 \mathrm{~km} \cdot \mathrm{s}^{-1}$. Columns include (left) the MD temperature profile $T_{\mathrm{MD}}$, (middle) the $\mathrm{HEq}$ temperature profile $T_{\mathrm{HEq}}$, and (right) the difference between MD and HEq profiles $T_{\mathrm{MD}}-T_{\mathrm{HEq}}$. Results at 50, 100, 250, and $1000 \mathrm{ps}$ are respectively shown in the rows from top to bottom. Contours are drawn in $150 \mathrm{~K}$ increments starting at $750 \mathrm{~K}$ for both $T_{\mathrm{MD}}$ and $T_{\mathrm{HEq}}$. 


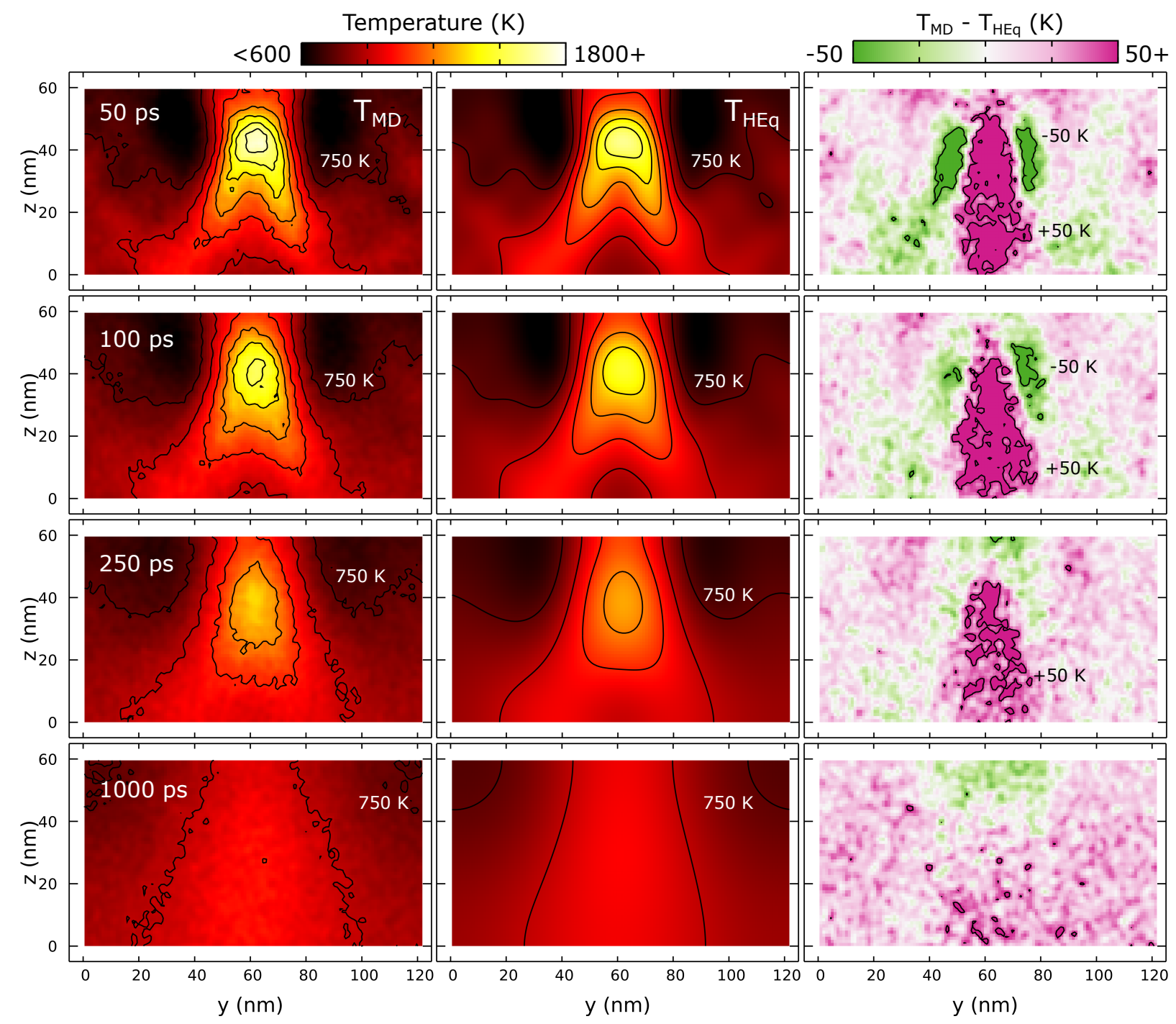

Figure 10: Comparison of temperature profiles from MD and an isotropic fitted HEq model for the case with $\mathbf{N}_{(001)}$ and $\left|\mathbf{U}_{\mathrm{p}}\right|=2.0 \mathrm{~km} \cdot \mathrm{s}^{-1}$. Columns include (left) the MD temperature profile $T_{\mathrm{MD}}$, (middle) the $\mathrm{HEq}$ temperature profile $T_{\mathrm{HEq}}$, and (right) the difference between MD and HEq profiles $T_{\mathrm{MD}}-T_{\mathrm{HEq}}$. Results at 50, 100, 250, and $1000 \mathrm{ps}$ are respectively shown in the rows from top to bottom. Contours are drawn in $150 \mathrm{~K}$ increments starting at $750 \mathrm{~K}$ for both $T_{\mathrm{MD}}$ and $T_{\mathrm{HEq}}$. 
average temperature of the system (see Figure 7). The HEq models are initialized with the MD temperature profile at $t=0 \mathrm{ps}$ and do not include heat source terms, so this amounts to an error in the final equilibrium temperature.

Errors in the fitted HEq models are largest at early times. Very similar trends were found for idealized 1D hot spot relaxation in crystalline TATB. ${ }^{45}$ It is reasonable that errors are largest when the gradients are at their largest and when the hot spot is most highly localized as this is precisely the regime where the assumptions underlying Fourier transport can break down. In particular, ballistic transport effects arise when the mean free path for energy carriers (phonons) is similar or smaller in size than the local problem dimension. This leads to an effective reduction in the conductivity.

Inspection of the temperature difference maps shows that the early time transport predicted by MD is slower than what the globally fit conductivity would suggest. The effective phonon mean free path estimated for crystalline TATB is on the order of a few nanometers, which would imply that a Fourier heat transfer model should hold even for nanoscale systems. ${ }^{16,45}$ This is broadly consistent with the present simulation results. As an upper bound, diamond and graphene have some of the largest phonon mean free paths, which can lead to significant reductions in the apparent thermal conductivity on sub-micron scales. ${ }^{64}$

\subsection{Comparison to Literature}

The anisotropic conductivity of TATB single crystal has been extensively characterized as a function of temperature, pressure, and molecular vacancy defect density ${ }^{15-17}$ through molecular simulation using the reverse non-equilibrium molecular dynamics (RNEMD) method ${ }^{66}$ and the TATB FF. Similar RNEMD calculations have been performed for the liquid at a number of distinct pressure-temperature states. ${ }^{44}$ These modeling results are largely consistent with recent determinations using density functional theory ${ }^{18}$ and other force fields. ${ }^{19}$ All reported experimental measurements for pure TATB were performed on polycrystalline pressed-powder samples in the 1970s and include the determinations of Faubion, ${ }^{67}$ Cornell 


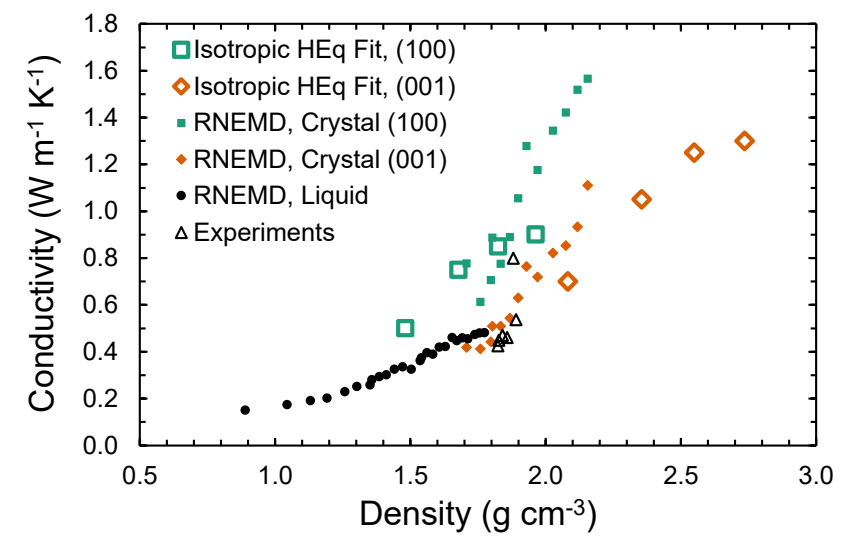

Figure 11: Comparison of isotropic TATB thermal conductivity values obtained from HEq fits to hot spot relaxation (open squares and diamonds) to TATB FF based RNEMD predictions in the literature (closed symbols) and experiments (open triangles). Previous crystal values include results for perfect crystals at various $(T, P)$ states and crystals with molecular vacancy defects near normal conditions.

and Johnson, ${ }^{68}$ and Baytos. ${ }^{69}$ To aid comparison across these diverse states and sample types, Figure 11 shows the present best-fit conductivity values along with literature values from MD simulations based on the TATB FF and from the available experiments.

The most striking feature of the plotted TATB conductivity values is their similarity and general increasing trend with density. This is despite substantial dissimilarity in the underlying states and contributing "measurement" techniques. Conductivity is largest within the TATB crystal layers along $\mathbf{N}_{(100)}$, but this only holds for perfect crystals. The values for the liquid state comprise an approximate lower bound owing to molecular-level disorder that limits the phonon mean free path. From kinetic theory considerations, ${ }^{70}$ the liquid conductivity is expected to depend linearly on density.

While isotropic HEq models perform well compared to anisotropic ones, the fitted isotropic conductivity depends on loading orientation as the average post-shock density depends on loading orientation. Anisotropy in the elastic tensor ${ }^{54}$ may partially explain this difference, as could anisotropy in the pore collapse mechanism. ${ }^{49}$ Zhao et al. ${ }^{43}$ predicted compression ratios of bulk shocked TATB crystal and found that the final density is approximately independent of shock orientation for $\mathbf{U}_{\mathrm{p}}=1 \mathrm{~km} \cdot \mathrm{s}^{-1}$. However, their compression ratio for 
shocks along $\mathbf{N}_{(001)}$ had large uncertainty and did not capture additional compression associated with plastic deformations. It is unclear whether bulk TATB single crystal exhibits an orientation-dependent post-shock density at other shock strengths or if the $\mathbf{N}_{(001)}$ direction is a special case with enhanced compressibility beyond what one would expect given the elastic properties of the material.

Many of the values obtained from fitting to hot spot relaxation simulations fall near the limiting liquid state values or else appear to comprise an extrapolation at densities above $2.0 \mathrm{~g} \cdot \mathrm{cm}^{3}$. This is likely the result of the highly disordered nature of sheared TATB within and around the hot spot core. ${ }^{31,32}$ While the $(T, P)$ states are below the predicted melting point of TATB, ${ }^{44}$ this sheared material is nonetheless more similar to the liquid state than to perfect crystal in terms of local structure. Disorder may also partly explain the relative success of the isotropic HEq model as applied to hot spots.

In absolute terms, the conductivity of TATB varies considerably from 0.2 to $1.6 \mathrm{~W} \cdot \mathrm{m}^{-1} \cdot \mathrm{K}^{-1}$ over a wide interval of density states. It also clear that material density near a collapsed pore can exhibit a wide range of density states depending on loading orientation and shock strength. Incorporation of a density-dependent conductivity in continuum-based simulations of hot spot dynamics appears warranted, either through an explicit density-dependent function or with a constant whose value is chosen specific to the simulation conditions. Obtaining effective conductivity values through fitting $\mathrm{HEq}$ models to $\mathrm{MD}$ predictions for hot spot relaxation provides a direct route to determine thermal transport coefficients at application-relevant conditions. This has a decided advantage in effectively averaging over potential ballistic effects and the manifold of temperature, pressure, and structural states in the vicinity of a hot spot, which would otherwise need to be independently characterized to arrive at an accurate model for the thermal physics of realistic hot spots.

The longstanding model for TATB hot spot criticality by Tarver et al. ${ }^{5}$ employed an isotropic temperature-dependent conductivity for unreacted TATB that decreased rapidly and approximately linearly from 0.88 to $0.42 \mathrm{~W} \cdot \mathrm{m}^{-1} \cdot \mathrm{K}^{-1}$ over a temperature range of 293 
to $673 \mathrm{~K}$. (Their model assumed $0.42 \mathrm{~W} \cdot \mathrm{m}^{-1} \cdot \mathrm{K}^{-1}$ above $673 \mathrm{~K}$.) While the conductivity is expected to decrease with increasing temperature at constant pressure, the present simulations suggest that this is more than offset by an increase in the conductivity with pressure. Given that the fitted hot spot conductivity is up to 3.1 times larger than the values of Tarver et al., even higher initial temperatures would be required for a hot spot of a given size to be considered critical in their model. In the case of RDX, Sakano et al. ${ }^{52}$ showed that small variations in the thermal conductivity parameter by less than $0.25 \mathrm{~W} \cdot \mathrm{m}^{-1} \cdot \mathrm{K}^{-1}$ altered predicted critical hot spot temperatures by over $100 \mathrm{~K}$. The range of conductivity values determined here for TATB hot spots $\left(0.80 \mathrm{~W} \cdot \mathrm{m}^{-1} \cdot \mathrm{K}^{-1}\right)$ is significantly larger than considered for RDX. The increasingly detailed understanding of thermal transport in explosives derived in recent years motivates a renewed assessment of hot spot criticality within the context of existing models.

\section{Conclusions}

We developed here a modeling approach to directly determine the thermal transport properties of explosive hot spots formed through shock-induced pore collapse with realistic structures and temperature fields. Molecular dynamics (MD) simulations form the basis of the approach and are used to sample pore collapse events and predict the ensuing thermal relaxation response out to nanosecond time scales. Diffusive heat equation (HEq) models are fit to the MD predictions treating the thermal conductivity as an adjustable parameter. Minimizing the root mean-square error between the MD prediction and HEq fit yields the effective thermal conductivity in the vicinity of a hot spot. This approach has well-defined approximations and yields information on the error in using HEq based models.

The approach is applied to characterize the thermal relaxation of quasi-2D hot spots in TATB, a layered molecular crystal that exhibits some of the largest and most anisotropic thermal conductivity values for an explosive near normal conditions. Our approach shows 
there is little difference between anisotropic and isotropic HEq models for TATB hot spots, which implies that treating the conductivity as isotropic is a reasonable approximation in grain- and component-scale models used for shock initiation studies. Thermal conductivity values for hot spot relaxation are generally lower than values for the perfect crystal at a given density. The conductivity is also found to be a strong function of density, which parametrically captures dependence on temperature, pressure, and material state and which varies by up to a factor of 2.6 under a range of conditions spanning from weak shocks to conditions typical of shock initiation. Conductivity values determined here for TATB hot spots are considerably larger than those used in a prior hot spot criticality study, which may significantly impact predictions for critical hot spot sizes.

Within the present HEq model approximations, the associated root-mean square errors of the fitted HEq models are within $\approx 5 \%$ of MD predictions. Many of these approximations might be relaxed in future work including: (1) incorporating heat sink and source terms for mechanical relaxation and/or chemistry; (2) treating the thermal conductivity as a fully populated tensor; (3) considering empirical forms for the thermal conductivity that depend on local variables such as density, temperature, pressure, shear strain, or other structural measures; (4) extending the modeling scheme to 3D system geometries. Primary advantages of the proposed modeling framework are that it provides a convenient foundation for determining the effective thermal conductivity for hot spot problems in explosives and other complex materials and directly yields information on reasonable approximations that might be taken in higher-level thermal physics models for those materials.

\section{Acknowledgement}

This work was supported by the Laboratory Directed Research and Development Program at Lawrence Livermore National Laboratory, project 18-SI-004 with Lara Leininger as P.I. Par-

tial support was received from the US Office of Naval Research, Multidisciplinary University 
Research Initiatives (MURI) Program, Contract: N00014-16-1-2557. Program managers: Clifford Bedford and Kenny Lipkowitz. Simulations were made possible by computing time granted to MPK through the LLNL Computing Grand Challenge, which is gratefully acknowledged. This work was performed under the auspices of the U.S. Department of Energy by Lawrence Livermore National Laboratory under Contract DE-AC52-07NA27344. It has been approved for unlimited release under document number LLNL-JRNL-823761.

\section{References}

(1) Handley, C. A.; Lambourn, B. D.; Whitworth, N. J.; James, H. R.; Belfield, W. J. Understanding the shock and detonation response of high explosives at the continuum and meso scales. Appl. Phys. Rev. 2018, 5, 011303.

(2) Mader, C. L. Initiation of detonation by the interaction of shocks with density discontinuities. Phys. Fluid 1965, 8, 1811.

(3) Field, J. E. Hot spot ignition mechanisms for explosives. Acc. Chem. Res. 1992, 25, 489.

(4) Hamilton, B. W.; Sakano, M. N.; Li, C.; Strachan, A. Chemistry under shock conditions. Annu. Rev. Mater. Res. 2021, 51.

(5) Tarver, C. M.; Chidester, S. K.; Nichols, A. L. Critical conditions for impact- and shock-induced hot spots in solid explosives. J. Phys. Chem. 1996, 100, 5794-5799.

(6) Wood, M. A.; Cherukara, M. J.; Kober, E. M.; Strachan, A. Ultrafast chemistry under nonequilibrium conditions and the shock to deflagration transition at the nanoscale. $J$. Phys. Chem. C 2015, 119, 22008.

(7) Springer, H. K.; Bastea, S.; Nichols III, A. L.; Tarver, C. M.; Reaugh, J. E. Modeling 
the effects of shock pressure and pore morphology on hot spot mechanisms in HMX. Propellants Explos. Pyrotech. 2018, 43, 805.

(8) Menikoff, R.; Sewell, T. D. Constituent properties of HMX needed for mesoscale simulations. Combust. Theory Model. 2002, 6, 103.

(9) Izvekov, S.; Chung, P. W.; Rice, B. M. Non-equilibrium molecular dynamics simulation study of heat transport in hexahydro-1,3,5-trinitro-s-triazine (RDX). Int. J. Heat Mass Trans. 2011, 54, 5623-5632.

(10) Sakano, M.; Hamilton, B.; Islam, M. M.; Strachan, A. Role of molecular disorder on the reactivity of RDX. J. Phys. Chem. C 2018, 122, 27032-27043.

(11) Kumar, G.; Van Gessel, F. G.; Elton, D. C.; Chung, P. W. Phonon lifetimes and thermal conductivity of the molecular crystal $\alpha$-RDX. MRS Adv. 2019, 4, 2191-2199.

(12) Perriot, R.; Powell, M. S.; Lazarz, J. D.; Bolme, C. A.; McGrane, S. D.; Moore, D. S.; Cawkwell, M. J.; Ramos, K. J. Pressure, temperature, and orientation dependent thermal conductivity of $\alpha$-1,3,5-trinitro-1,3,5-triazinane ( $\alpha$-RDX). arXiv 2021,

(13) Long, Y.; Chen, J.; Liu, Y. G.; Nie, F. D.; Sun, J. S. A direct method to calculate thermal conductivity and its application in solid HMX. J. Phys.: Condens. Matter 2010, 22, 185404.

(14) Rezvan, C.; Kroonblawd, M. P.; Pereverzev, A.; Sewell, T. A molecular dynamics simulation study of thermal conductivity anisotropy in $\beta$-octahydro-1,3,5,7-tetranitro1,3,5,7-tetrazocine ( $\beta$-HMX). Modell. Simul. Mater. Sci. Eng. 2020, 28, 025008.

(15) Kroonblawd, M. P.; Sewell, T. D. Theoretical determination of anisotropic thermal conductivity for crystalline 1,3,5-triamino-2,4,6-trinitrobenzene (TATB). J. Chem. Phys. 2013, 139, 074503. 
(16) Kroonblawd, M. P.; Sewell, T. D. Theoretical determination of anisotropic thermal conductivity for initially defect-free and defective TATB single crystals. J. Chem. Phys. 2014, 141, 184501.

(17) Kroonblawd, M. P.; Sewell, T. D. Predicted anisotropic thermal conductivity for crystalline 1,3,5-triamino-2,4,6-trinitobenzene (TATB): Temperature and pressure dependence and sensitivity to intramolecular force field terms. Propellants Explos. Pyrotech. $41,502-513$.

(18) Long, Y.; Chen, J. Theoretical study of the phonon-phonon scattering mechanism and the thermal conductive coefficients for energetic material. Phil. Mag. 2017, 97, 25752595.

(19) Fan, H.; Long, Y.; Ding, L.; Chen, J.; Nie, F.-D. A theoretical study of elastic anisotropy and thermal conductivity for TATB under pressure. Comp. Mat. Sci. 2017, 131, 321332.

(20) Chernatynskiy, A.; Phillpot, S. R. Phonon-mediated thermal transport: Confronting theory and microscopic simulation with experiment. Curr. Opin. Solid State Mater. Sci. 2013, 17, 1-9.

(21) Cahill, D. G.; Braun, P. V.; Chen, G.; Clarke, D. R.; Fan, S.; Goodson, K. E.; Keblinski, P.; King, W. P.; Mahan, G. D.; Majumdar, A. et al. Nanoscale thermal transport II. 2003-2012. Appl. Phys. Rev. 2014, 1, 011305.

(22) Minnich, A. J.; Johnson, J. A.; Schmidt, A. J.; Esfarjani, K.; Dresselhaus, M. S.; Nelson, K. A.; Chen, G. Thermal conductivity spectroscopy technique to measure phonon mean free paths. Phys. Rev. Lett. 2011, 107, 095901.

(23) Minnich, A. J. Determining phonon mean free paths from observations of quasiballistic thermal transport. Phys. Rev. Lett. 2012, 109, 205901. 
(24) Dettori, R.; Ceriotti, M.; Hunger, J.; Colombo, L.; Donadio, D. Energy relaxation and thermal diffusion in infrared pump-probe spectroscopy of hydrogen-bonded liquids. $J$. Phys. Chem. Lett. 2019, 10, 3447-3452.

(25) Stukowski, A. Visualization and analysis of atomistic simulation data with OVITO the Open Visualization Tool. Model. Simul. Mater. Sci. Eng. 2010, 18, 015012, OVITO is available at https://www.ovito.org.

(26) Falk, M. L.; Langer, J. S. Dynamics of viscoplastic deformation in amorphous solids. Phys. Rev. E: Stat., Nonlin., Soft Matter Phys. 1998, 57, 7192-7205.

(27) Cawkwell, M. J.; Sewell, T. D.; Zheng, L.; Thompson, D. L. Shock-induced shear bands in an energetic molecular crystal: Application of shock-front absorbing boundary conditions to molecular dynamics simulations. Phys. Rev. B: Condens. Matter Mater. Phys. 2008, 78, 014107.

(28) Cawkwell, M. J.; Ramos, K. J.; Hooks, D. E.; Sewell, T. D. Homogeneous dislocation nucleation in cyclotrimethylene trinitramine under shock loading. J. Appl. Phys. 2010, $107,063512$.

(29) Eason, R. M.; Sewell, T. D. Shock-induced inelastic deformation in oriented crystalline pentaerythritol tetranitrate. J. Phys. Chem. C 2012, 116, 2226-2239.

(30) Khan, M.; Picu, C. R. Shear localization in molecular crystal cyclotetramethylenetetranitramine ( $\beta$-HMX): Constitutive behavior of the shear band. J. Appl. Phys. 2020, 128, 105902.

(31) Kroonblawd, M. P.; Fried, L. E. High explosive ignition through chemically activated nanoscale shear bands. Phys. Rev. Lett. 2020, 124, 206002.

(32) Hamilton, B. W.; Kroonblawd, M. P.; Li, C.; Strachan, A. A hotspot's better half: 
Non-equilibrium intra-molecular strain in shock physics. J. Phys. Chem. Lett. 2021, 12, 2756-2762.

(33) Austin, R. A.; Barton, N. R.; Reaugh, J. E.; Fried, L. E. Direct numerical simulation of shear localization and decomposition reactions in shock-loaded HMX crystal. J. Appl. Phys. 2015, 117, 185902.

(34) Islam, M. M.; Strachan, A. Role of dynamical compressive and shear loading on hotspot criticality in RDX via reactive molecular dynamics. J. Appl. Phys. 2020, 128, 065101.

(35) Cady, H. H.; Larson, A. C. The crystal structure of 1,3,5-triamino-2,4,6-trinitrobenzene. Acta Crystallogr. 1965, 18, 485-496.

(36) Suemune, Y. Thermal conductivity of some ferroelectric crystals with hydrogen bonds. J. Phys. Soc. Japan 1967, 22, 735-743.

(37) Slack, G. A. Thermal conductivity of ice. Phys. Rev. B: Condens. Matter Mater. Phys. 1980, 22, 3065-3071.

(38) Mathew, N.; Sewell, T. D. Generalized stacking fault energies in the basal plane of triclinic molecular crystal 1,3,5-triamino-2,4,6-trinitrobenzene (TATB). Phil. Mag. 2015, 95, 424-440.

(39) Lafourcade, P.; Denoual, C.; Maillet, J.-B. Dislocation core structure at finite temperature inferred by molecular dynamics simulations for 1,3,5-triamino-2,4,6-trinitrobenzene single crystal. J. Phys. Chem. C 2017, 121, 7442-7449.

(40) Lafourcade, P.; Denoual, C.; Maillet, J.-B. Irreversible deformation mechanisms for 1,3,5-triamino-2,4,6-trinitrobenzene single crystal through molecular dynamics simulations. J. Phys. Chem. C 2018, 122, 14954-14964.

(41) Steele, B. A.; Stavrou, E.; Prakapenka, V. B.; Kroonblawd, M. P.; Kuo, I.-F. W. High-pressure equation of state of 1,3,5-triamino-2,4,6-trinitrobenzene: Insights into 
the monoclinic phase transition, hydrogen bonding, and anharmonicity. J. Phys. Chem. A 2020, 124, 10580-10591.

(42) Lafourcade, P.; Denoual, C.; Maillet, J.-B. Mesoscopic constitutive law with nonlinear elasticity and phase transformation for the twinning-buckling of TATB under dynamic loading. Phys. Rev. Mat. 2019, 3, 053610.

(43) Zhao, P.; Kroonblawd, M.; Mathew, N.; Sewell, T. Strongly Anisotropic Thermomechanical Response to Shock Wave Loading in Oriented Samples of the Triclinic Molecular Crystal 1,3,5-Triamino-2,4,6-Trinitrobenzene (TATB). ChemRxiv 2021,

(44) Mathew, N.; Kroonblawd, M. P.; Sewell, T.; Thompson, D. L. Predicted melt curve and liquid-state transport properties of TATB from molecular dynamics simulations. Mol. Sim. 2018, 44, 613-622.

(45) Kroonblawd, M. P.; Sewell, T. D. Anisotropic relaxation of idealized hot spots in crystalline 1,3,5-triamino-2,4,6-trinitrobenzene (TATB). J. Phys. Chem. C 2016, 120, $17214-17223$.

(46) Eason, R. M.; Sewell, T. D. Molecular dynamics simulations of the collapse of a cylindrical pore in the energetic material $\alpha$-RDX. J. Dyn. Behav. Mater. 2015, 1, 423-438.

(47) Long, Y.; Chen, J. Theoretical study of the defect evolution for molecular crystal under shock loading. J. Appl. Phys. 2019, 125, 065107.

(48) Li, C.; Hamilton, B. W.; Strachan, A. Hotspot formation due to shock-induced pore collapse in 1,3,5,7-tetranitro-1,3,5,7-tetrazoctane (HMX): Role of pore shape and shock strength in collapse mechanism and temperature. J. Appl. Phys. 2020, 127, 175902.

(49) Zhao, P.; Lee, S.; Sewell, T.; Udaykumar, H. S. Tandem molecular dynamics and continuum studies of shock-induced pore collapse in TATB. Propellants Explos. Pyrotech. 2020, 45, 196-222. 
(50) Zhou, T.; Song, H.; Liu, Y.; Huang, F. Shock initiated thermal and chemical responses of HMX crystal from ReaxFF molecular dynamics simulation. Phys. Chem. Chem. Phys. 2014, 16, 13914-13931.

(51) Lee, K.; Joshi, K.; Chaudhuri, S.; Stewart, D. S. Mirrored continuum and molecular scale simulations of the ignition of high-pressure phases of RDX. J. Chem. Phys. 2016, $144,184111$.

(52) Sakano, M. N.; Hamed, A.; Kober, E. M.; Grilli, N.; Hamilton, B. W.; Islam, M. M.; Koslowski, M.; Strachan, A. Unsupervised learning-based multiscale model of thermochemistry in 1,3,5-trinitro-1,3,5-triazinane (RDX). J. Phys. Chem. A 2020, 124, 9141-9155.

(53) Plimpton, S. Fast parallel algorithms for short-range molecular dynamics. J. Comput. Phys. 1995, 117, 1, LAMMPS is available at http://lammps.sandia.gov.

(54) Bedrov, D.; Borodin, O.; Smith, G. D.; Sewell, T. D.; Dattelbaum, D. M.; Stevens, L. L. A molecular dynamics simulation study of crystalline 1,3,5-triamino2,4,6-trinitrobenzene as a function of pressure and temperature. J. Chem. Phys. 2009, $131,224703$.

(55) Mathew, N.; Sewell, T. D.; Thompson, D. L. Anisotropy in surface-initiated melting of the triclinic molecular crystal 1,3,5-triamino-2,4,6-trinitrobenzene: A molecular dynamics study. J. Chem. Phys. 2015, 143, 094706.

(56) Ryckaert, J.-P.; Ciccotti, G.; Berendsen, H. J. Numerical integration of the cartesian equations of motion of a system with constraints: molecular dynamics of n-alkanes. $J$. Comp. Phys. 1977, 23, $327-341$.

(57) Andersen, H. C. Rattle: A "velocity" version of the shake algorithm for molecular dynamics calculations. J. Comp. Phys. 1983, 52, $24-34$. 
(58) Wolf, D.; Keblinski, P.; Phillpot, S. R.; Eggebrecht, J. Exact method for the simulation of Coulombic systems by spherically truncated, pairwise r-1 summation. J. Chem. Phys. 1999, 110, 8254 .

(59) Holian, B. L.; Lomdahl, P. S. Plasticity induced by shock waves in nonequilibrium molecular-dynamics simulations. Science 1998, 280, 2085-2088.

(60) Nosé, S. A unified formulation of the constant temperature molecular dynamics methods. J. Chem. Phys. 1984, 81, 511.

(61) Hoover, W. G. Canonical dynamics: Equilibrium phase-space distributions. Phys. Rev. A: At., Mol., Opt. Phys. 1985, 31, 1695.

(62) Kroonblawd, M. P.; Mathew, N.; Jiang, S.; Sewell, T. D. A generalized crystal-cutting method for modeling arbitrarily oriented crystals in 3D periodic simulation cells with applications to crystal-crystal interfaces. Comput. Phys. Commun. 2016, 20\%, 232.

(63) Zhao, S.; Germann, T. C.; Strachan, A. Atomistic simulations of shock-induced alloying reactions in Ni-Al nanolaminates. J. Chem. Phys. 2006, 125, 164707.

(64) Christenson, J. G.; Kroonblawd, M. P.; Austin, R. A.; Fried, L. E.; Phillips, R. J. Simulating transient heat transfer in graphene at finite Knudsen number via the Boltzmann transport equation and molecular dynamics. Phys. Rev. B: Condens. Matter Mater. Phys. 2020, 102, 205406.

(65) Asmar, N. H. Partial differential equations with Fourier series and boundary value problems; Pearson Prentice Hall: Upper Saddle River, NJ, 2005.

(66) Müller-Plathe, F. A simple nonequilibrium molecular dynamics method for calculating the thermal conductivity. J. Chem. Phys. 1997, 106, 6082-6085.

(67) Faubion, B. D. Thermal conductivity of TATB and TATB blends by differential scanning calorimetry; 1976; Report No. MHSMP-76-30C. 
(68) Cornell, R. H.; Johnson, G. L. Measuring thermal diffusivities of high explosives by the flash method; 1978; Report No. UCRL-52565.

(69) Baytos, J. F. Specific heat and thermal conductivity of explosives, mixtures, and plasticbonded explosives determined experimentally; 1979; Report No. LA-8034-MS.

(70) Bird, R. B.; Stewart, W. E.; Lightfoot, E. N. Transport phenomena; Wiley: New York, NY, 2007. 